\title{
Transfection of STAT3 Overexpression Plasmid Mediated by Recombinant Lentivirus Promotes Differentiation of Bone Marrow Mesenchymal Stem Cells Into Neural Cells in Fetal Rats With Spina Bifida Aperta
}

mingyu Jiang ( $\sim 94178268 @ q q . c o m$ )

First Affiliated Hospital of Harbin Medical University https://orcid.org/0000-0002-0359-0064

Ji-cheng Dai

First Affiliated Hospital of Harbin Medical University

Ming-ying Yin

Fourth Affiliated Hospital of Harbin Medical University

Ming-yong Ren

First Affiliated Hospital of Harbin Medical University

\section{Research}

Keywords: bone marrow mesenchymal stem cells, spina bifida aperta, signal transducer and activator of transcription-3, lentivirus transfection, nerve cells

Posted Date: March 4th, 2021

DOI: https://doi.org/10.21203/rs.3.rs-264087/v1

License: (c) (i) This work is licensed under a Creative Commons Attribution 4.0 International License.

Read Full License 


\section{Abstract \\ Background}

To investigate the influence of signal transducer and activator of transcription-3 (STAT3) on spinal cord tissue grafts of rat fetuses with spina bifida aperta. In particular, we wished to determine if STAT3 overexpression plasmid transfection would increase survival of spinal cord transplantation to improve therapeutic efficiency.

\section{Methods}

The fetal rat model of spina bifida aperta was established by retinoic acid and treated by microsurgical injection of stem cells. They were divided into blank control group $(n=12)$, negative control group $(n=10)$ and experimental group $(n=11)$. The optical density (OD) value of BMSCs viability was determined by Cell Counting Kit-8 (CCK-8). The expression of STAT3, pSTAT3, nerve markers (Glial fibrillary acidic protein, Neuron-specific enolase, Neurofilament and Nestin) and apoptosis related factors (Caspase 8 and Bcl-2) were tested by the method of real-time PCR and Western blot. All statistical analyses were performed using SPSS version 20.0 software. One-way analysis of variance was used to analyze differences among three or more groups. The data are presented as the mean \pm standard deviation from at least three independent experiments. $P<0.05$ was considered statistically significant.

\section{Results}

OD value in experimental group was the highest at 8 hours after transplantation by CCK-8, and then gradually decreased, which was statistically significant compared with blank control group and negative control group $(P<0.05)$. There was no statistical difference in OD peak time and value between blank control group and negative control group. The expression levels of pSTAT3, Glial fibrillary acidic protein, Neuron-specific enolase, Neurofilament and Nestin in experimental group were remarkably higher than those in blank control group and negative control group $(P<0.05)$, but STAT3 expression in experimental group were statistically decreased $(P<0.05)$. The relative expression levels of Caspase-8 and Bcl-2 in experimental group were tremendously lower than those in blank control group and negative control group $(P<0.05)$.

\section{Conclusion}

Transfection of recombinant lentivirus mediated STAT3 overexpression plasmid with BMSCs can improve the efficiency of transforming into neural cells and provide new seed cells for the treatment of congenital spina bifida aperta. 


\section{Introduction}

Neural tube defects (NTDs) can lead to serious birth defects in children. At present, it is believed that the main mechanism of NTDs is abnormal closure of neural tube. Among them, congenital spina bifida aperta (SBA) is the most common [1]. The etiology and pathogenesis of SBA are not clear, and it is generally believed that a variety of abnormal gene regulation and environmental harmful factors take part in the process of embryonic development [2]. Apoptosis is considered to be an important event in the formation, development and maturity of nervous system [3]. Many data show that neural tube malformation in children is related to neuronal apoptosis, but the exact mechanism is still unclear. Developmental biology research shows that apoptosis occurs at a specific time and place during the normal development of embryo. If this fine regulated, programmed cell death changes, it may lead to various types of congenital malformations [4].

At present, there is no effective treatment for SBA in children. The severe malformations die in the embryonic period. Even if the patient with slight malformation can survive, most of them show neurological dysfunction in different degree, including limb paralysis, defecation dysfunction, paraplegia, etc [5]. It seriously affects the growth and development of children, and life quality in the future. However, in recent years, the technology of stem cell transplantation has brought vitality and hope for the treatment of congenital nervous system (NS) malformation. Bone marrow mesenchymal stem cells (BMSCs), as a kind of adult stem cells, have self-renewal and multi-directional differentiation potentials. Under the action of some physical, chemical factors and cytokines, BMSCs can differentiate into neural cells, osteoblasts, chondrocytes, myoblasts, adipocytes and other kinds of cells [6, 7]. It can also differentiate into mesoderm stromal cells, ectoderm or endoderm cells under certain conditions or microenvironment, which plays an important role in the treatment of the nervous system degenerative diseases, stress injury and congenital malformation. This technology is widely used in gene therapy and bioremediation $[8,9]$. Because of simple material, easy separation and purification, BMSCs still have strong differentiated ability after several passages, and they derive from their own without matching, so immune exclusion is rare. Therefore, BMSCs can be used as an important source of seed cells in tissue engineering. BMSCs transplantation is expected to be a new method to treat children's SBA.

STAT3, as an important member of STAT family, was found and purified in 1994 as an acute stage response factor (aprf) [10]. STAT3 can be activated by many cytokines and growth factors, such as IL-6, LIF, CNTF, Onco-statin M and EGF. STAT3 signal transduction pathway is activated by recognizing the receptor complex, which formed by glycoprotein gp130 on cell surface and other molecules such as LIFR and CNTFR. Some scholars believe that the coordination between STAT3 and MAPK signaling pathway plays an active role in maintaining self-renewal and differentiation of stem cells [11]. STAT and MAPK family are participants of important signal transduction pathways within cells, both of them can transmit stimulation from extracellular to nuclear, and participate in a series of physiological processes such as cell growth, development, differentiation and apoptosis [12]. In addition, STAT3 protein not only participates in many physiological processes in the development of nervous system, but also regulates the survival and repair of nerve cells by neuroregulatory cytokines. Caspase-8 is a major member of 
caspase family, as a group of aspartic acid specific cysteine protease, which plays a key role in the execution of apoptosis and determines cell morphological and physicochemical change [13].Meanwhile, $\mathrm{Bcl}-2$ directly involves in early initiation, signal transmission and late effect of apoptosis [14]. Once the expression of caspase- 8 and $\mathrm{Bcl}-2$ is abnormal, it can lead to apoptosis and proliferation disorders, thus inducing occurrence of some nervous system diseases [15].

In this experiment, BMSCs were isolated and purified by using the whole bone marrow adherence method, STAT3 overexpression plasmid was transfected into BMSCs by recombinant lentivirus, and the fetal rat model of SBA was established by retinoic acid. The fetal rats were treated with BMSC transplantation by combination of fetal surgery and microsurgery injection. The expression of STAT3, pSTAT3, nerve markers (GFAP, NSE, NF and nestin), apoptosis related factors (Caspase 8 and Bcl-2) in the spinal cord of fetal rats was observed, in order to explore the role of STAT3 in the transplantation therapy of SBA.

\section{Materials And Methods}

\section{Ethical approval}

All experimental procedures for animal usage were in accordance with the guidelines for care and use of laboratory animals and were approved by the ethical committee of Harbin Medical University.

\section{Animal models}

Ten- to twelve-week-old adult wistar rats, weighting 250-300g, were selected, which obtained from Animal Research Center of Harbin Medical University (Harbin, China).

Virginal female rats were mated with males of the same strain and the date of vaginal plug detection was designated EO. Spina bifida aperta were induced with a single intragastric retinoic acid $(140 \mathrm{mg} / \mathrm{kg}$ body weight; Sigma) administration on E10 as previously described (Cai, W.; Zhao, H.; Guo, J.; Li, Y.; Yuan, Z.; Wang, W. Retinoic acid-induced lumbosacral neural tube defects: myeloschisis and hamartoma. Childs Nerv. Syst. 23(5):549-554; 2007).

\section{Isolation and transfection of bone marrow mesenchymal stem cells (BMSCs)}

The cells were obtained from the femur of 4-week-old wistar rats. Rat bone marrow was flushed with $20 \mathrm{ml} \mathrm{DMEM} / \mathrm{F} 12$ medium and 10\% fetal bovine serum (FBS), and cell suspension was isolated as BMSCs through the method of bone marrow adherence for the primary generation (P0), then cells were seeded in a tissue culture incubator at $37{ }^{\circ} \mathrm{C}$ under $5 \% \mathrm{CO}$. Cells were passaged $5-7$ days to achieve the desired dose until passage 6 (P6). The BMSCs of P6 were plated in a 6-well plate to grow to $90 \%$ confluent before transfection (about $1 \times 10^{5}$ cells/well). Twenty four hours before transplantation, BMSCs were transfected with recombinant lentivirus and STAT3 overexpression plasmid (SinoGenoMax.Co., Ltd, Beijing, China)(100pfu/1cell). The concentration of BMSCs suspension for transplantation is about 20000 cells $/ \mu$ l. 


\section{Identification of bone marrow mesenchymal stem cells by flow cytometry}

The surface proteins of CD34, CD45, CD73 and CD90 were determined by flow cytometry. The extraction, identification and purity characterization of BMSCs provide scientific basis.

\section{BMSCs viability was determined by CCK-8}

First of all, the cell count was improved. The cells were inoculated in the 96 well 4 plate, and the number of cells in each well was maintained at least $5 \times 10^{4}$. At the same time, two multiple wells were set and further incubated in the cell incubator at $37^{\circ} \mathrm{C} .10 \mu$ I CCK-8 solution (TaKaRa Bio Inc, Japan) was added to each well at $4,8,12,16,20,24,48$ and 72 hours. The optical density (OD) value was detected at 450 $\mathrm{nm}$.

\section{Transplantation procedure and animal group}

A total of seventy-two female wistar rats were subjects of this study. Animals had free access to food and water throughout the study. On embryonic day (E15, E16 and E17), all the surgeries were done under anesthesia with dose $(40 \mathrm{mg} / \mathrm{kg})$ of pentobarbital sodium (Sigma) and atropine $(0.25 \mathrm{mg} / \mathrm{ml}$, Sigma) intraperitoneally. Then the pregnant rats underwent midline laparotomy to expose the uterine horns. To relieve uterine spasm, the uterus was covered with wet gauze immersed with warm physiologic saline and atropine $(0.1 \mathrm{mg} / \mathrm{kg})$ was given intraperitoneally. Under the microscope the position of lumbosacral spine of fetus was identified through the wall of uterus. Then 7-0-nylon purse-string suture and a small incision were made on the wall of uterus. The amniotic sac was opened and the defective region of spinal cord was exposed. BMSCs with STAT3 overexpression plasmid transfection $(0.2 \mu \mathrm{l} /$ injection site) was injected into the defective region of spinal cord with a micropipette (internal tip diameter $100 \mu \mathrm{m}$ ) connected to a Hamilton syringe (experimental group). Micropipettes for injection were made from borosilicate glass capillaries (model GD-1; Narishige Scientific Instruments, Tokyo, Japan) by using a micropipette puller (model PB-7; Narishige Scientific Instruments, Tokyo, Japan). After BMSCs injection, the fetuses were returned to the uterus, and the wound of the uterus was closed. The same volume of BMSCs without recombinant lentivirus transfection (blank control group) and only recombinant lentivirus transfection without STAT3 overexpression plasmid (negative control group). In average, 2 to 3 fetuses could be injected in one dam without compromising the survival of the fetuses. The pregnant rats recovered from the anesthesia within 1 hour and were returned to their home cage. The pregnant rats on E18, E19, E19.5 and E20 as needed were reanesthetized, and the fetuses that had been transplanted were harvested by cesarean section. Each fetus was inspected for the presence of external malformations.

\section{Real-time polymerase chain reaction analysis}

Total RNA was isolated using TRIzol reagent (Invitrogen Co, USA) according to the manufacturer's instructions, and cDNA was generated through reverse transcription using the PrimeScript RT Master Mix kit (TaKaRa Bio Inc, Japan). Equal amounts of cDNA were diluted and amplified through real-time polymerase chain reaction (PCR) using All-in-one qPCR Mix (TaKaRa Bio Inc) in a $20 \mu$ reaction volume 
containing $10 \mu \mathrm{l}$ of $2 \times$ All-in-one qPCR Mix (TaKaRa Bio Inc), $1 \mu \mathrm{l}$ of $2 \mathrm{mmol} / \mathrm{L}$ forward primer, $1 \mu \mathrm{l}$ of 2 $\mathrm{mmol} / \mathrm{L}$ reverse primer, $1 \mu \mathrm{l}$ of $\mathrm{CDNA}$, and $6 \mu \mathrm{l}$ of nuclease-free water. After an initial denaturation step for $10 \mathrm{~min}$ at $95^{\circ} \mathrm{C}$, the conditions for cycling were 40 cycles of $10 \mathrm{~s}$ at $95^{\circ} \mathrm{C}, 20 \mathrm{~s}$ at $60^{\circ} \mathrm{C}$, and $15 \mathrm{~s}$ at $72^{\circ} \mathrm{C}$. For the normalization of each sample, glyceraldehyde 3-phosphate dehydrogenase (GAPDH, TaKaRa Bio Inc) primers were used to measure the amount of GAPDH cDNA. The primers used are listed as Table 1. The relative fold-change in the target gene cDNA was determined using the $2^{-\triangle \Delta C t}$ method.

\section{Western blotting analysis}

The protein levels in spinal cord tissues were detected by Western blotting. Briefly, the tissues from different groups were lysed by Radio-Immunoprecipitation Assay lysis buffer (Beyotime Bio Inc, Shanghai, China). The protein concentrations were determined by BCA protein estimation kit (Beyotime Bio Inc). Then, equal amounts of protein samples were separated by sodium dodecyl sulphatepolyacrylamide gel electrophoresis (Beyotime Bio Inc), and transferred to polyvinylidene difluoride membranes (Beyotime Bio Inc). After blocking with 5\% non-fat dry milk, the membranes were incubated with primary antibodies at $4^{\circ} \mathrm{C}$ overnight. The primary antibodies used in this study were as follows: STAT3 (Santa Cruz Bio Inc, USA), caspase-8 (Santa Cruz Bio Inc, USA), and GAPDH (Santa Cruz Bio Inc, USA). Then the corresponding secondary antibodies conjugated with horseradish peroxidase were added. ECL detection reagent (7 Sea Biotech, Shanghai, China) was used to visualize the blots. The results were quantified with Gel-Pro-Analyzer (Media Cybernetics, USA).

\section{Statistical analysis}

All statistical analyses were performed using SPSS version 20.0 software (SPSS, Inc., Chicago). The unpaired Student $t$ test was used to analyze differences between only two value sets. One-way analysis of variance was used to analyze differences among three or more groups. The data are presented as the mean \pm standard deviation from at least three independent experiments. $P<0.05$ was considered statistically significant.

\section{Result}

BMSCs were successfully identificated with flow cytometry method in wistar rats before transplantation

Four kinds of BMSC surface markers at P0-6 were detected by flow cytometry. The results showed that CD73 and CD90 were positive, CD34 and CD45 were negative (Figure 1A). Among them, CD73 and CD90 were fibrin and ribosomal receptors on the surface of BMSC, which were non-hematopoietic cell origin. CD34 and CD45 were the surface receptors of endothelial cells and hematopoietic cells, which indicated that BMSCs from wistar rats have been successfully isolated.

BMSCs proliferation activity were determined by CCK-8 test and the STAT3 plasmid was successfully transfected before transplantation 
At $4,8,12,16,20,24,48$ and 72 hours as time nodes, BMSCs proliferation activity at P0, P1-3, P4-6 in blank control group, negative control group and experimental group was detected before transplantation. The results showed that there was no significant difference in the number of living cells among the three groups at the time point $(P>0.05)$, which suggested that lentivirus transfection has no significant effect on the proliferation of BMSCs ( Figure 1B, C and D). STAT3 mRNA expression in experimental group increased more statistically than that in blank control group and negative control group $(P<0.05)$, which demonstrated that STAT3 overexpression plasmid transfection was successfully constructed ( Figure 1E, $F$ and $G$ ).

\section{Survival of rat fetuses in each group after BMSCs transplantation}

220 fetuses of 72 pregnant rats were BMSCs transplanted, 5 pregnant rats died after transplantation on the 18th day, the survival rate of transplanted pregnant rats was $93 \%$ (67/72). The pregnant, transplanted rats were killed on E18, E19, E19.5 and E20, then transplanted fetuses with SBA were harvested for lumbosacral spinal sample collection. 70 cases arised from MSCs without recombinant lentivirus transfection (blank control group); 74 cases from MSCs with STAT3 overexpression plasmid transfection (experimental group), 76 cases with only recombinant lentivirus transfection (negative control group).

\section{BMSCs combined with STAT3 overexpression plasmid transfection can improve the success rate of transplantation}

Before transplantation, The purity of BMSCs was about enriched from $35 \pm 1.02 \%$ at $\mathrm{P} 0,34 \pm 1.16 \%$ at $\mathrm{P} 1-3$, and $33 \pm 1.22 \%$ from P4 to P6 in blank control group and negative control group. However, as BMSCs combined with STAT3 overexpression plasmid transfection processed as above. The purity of BMSCs was enriched from $75 \pm 1.13 \%$ at $\mathrm{P0}, 87 \pm 1.29 \%$ from $\mathrm{P} 1$ to $\mathrm{P} 3$, and $73 \pm 1.07 \%$ from $\mathrm{P} 4$ to $\mathrm{P} 6$ when $\mathrm{CD} 73$ and CD90 expression was also tested, which was statistically significant $(P=0.031,0.023$ and 0.037 , compared with blank control group and negative control group, respectively) (Figure 2A). P1-3 BMSCs combined with STAT3 overexpression plasmid transfection has a better purity than P0 and P4-6. Then we transplanted P1-3 BMSCs into each group of rat fetuses on E15, E16 and E17 to examine whether STAT3 overexpression plasmid transfection may affect cell survival ratio. Green fluorescent protein (GFP) was labeled to BMSCs and positive cell numbers were counted on E19 after transplantation. The overall survival rates of BMSCs in experimental group was found to be $36 \pm 1.08 \%$ on E15, $46 \pm 0.72 \%$ on E16 and $35 \pm 0.93 \%$ on E17, which was a little higher than that of blank control group and negative control group, and statistically significant $(P=0.029,0.021$ and 0.033$)$. Better survival after STAT3 overexpression plasmid transfection was achieved in the experimental groups performed on later development stages (E16) as compared with E15 and E17 group, but it did not reach a statistical significance ( $P=0.65 \mathrm{E} 15$ versus E16; $P=0.32 \mathrm{E} 17$ versus E16) (Figure 2B). Secondly, we wanted to detect whether different BMSC passages transplanted after STAT3 overexpression plasmid transfection could interpose survival ratio, and three groups were divided on E16 transplanation from rat fetuses: P0, P1-3 and to P4-6. BMSCs

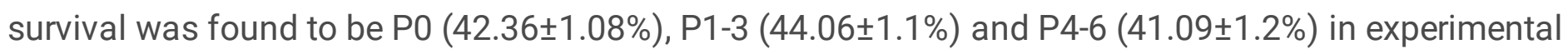
group from E19 transplanted fetuses, which showed a notable increase compared with blank control 
group and negative control group, again the differences reached a statistical significance $(P=0.023$, 0.021 and 0.026 , respectively) (Figure 2C). Thirdly, we evaluated the effects of injected cell number on survival ratio after P1-3 BMSCs with STAT3 overexpression plasmid transfection on E16 transplanation from rat fetuses. Medium injection group (2000-4000 cells/injection/spinal cord) was remarkably different from the change that occurred in low injection group (1000-1999 cells/injection/spinal cord) and high injection group (4001-6000 cells/injection /spinal cord) on E19 transplanted fetuses, which reached a statistical significance $(P=0.026,0.022$ and 0.029 , respectively) (Figure 2D).

\section{CCK-8 test was used to detect cell proliferation activity after BMSCs transplantation again}

At $4,8,12,16,20$ and 24 hours as time nodes, the proliferation activity of P0-6 BMSCs in blank control group, negative control group and experimental group was detected, and OD value of living cells in each group was measured. The results showed that OD value in experimental group was the highest at 8 hours on E19 after E16 transplantation, and then gradually decreased, which was significantly different from blank control group and negative control group $(P<0.05)$ (Figure 3A-C). However, there was no significant difference in OD peak time and value between blank control group and negative control group.

\section{Analysis of STAT3 and pSTAT3 expression in spinal cord of each group after BMSCs transplantation}

The developmental change in pSTAT3 expression of experimental group from P1-3 BMSCs transplantation was notably different from the change that occurred in blank control group and negative control group on E18, E19, E19.5 and E20, especially at embryonic day 19 (E19). Both pSTAT3 mRNA and protein levels were significantly increased in experimental group from E18 to E20, and E19 reached the peak (Figure 4A, B and C). Overall, the expression of STAT3 mRNA and protein levels in experimental group gradually decreased with embryonic development between E18 and E19, E19 dropped down to bottom. Then From E18 to E20, pSTAT3 and STAT3 in mRNA and protein levels kept steady level in blank control group and negative control group, which statistical decreased than that in experimental group $(P<$ 0.05) (Figure 4D, E and F).

\section{Analysis of GFAPINSEnNF and Nestin expression in spinal cord of each group after BMSCs transplantation}

The relative expression of GFAP mRNA in blank control group, negative control group and experimental group was statistically different from P1-3 BMSCs transplantation on E16. The level of GFAP mRNA in experimental group was significantly higher than that in blank control group and negative control group on E19 transplanted fetuses $(P<0.05)$. At the same time, the relative expression trend of NSE, NF and Nestin mRNA in each group was basically consistent with above (Figure $5 \mathrm{~A}$ ). In addition, the protein levels of GFAP, NSE, NF and Nestin using Western Blot were identical with real-time PCR (Figure 5B,C).

\section{Analysis of Caspase-8 and Bcl-2 expression in spinal cord of each group after MSCs transplantation}

Caspase-8 and Bcl-2 as apoptosis gene play an important role in the development of spina bifida aperta. Next we studied the apoptosis of whole spinal column after BMSCs transplantation with STAT3 
overexpression plasmid transfection from caspase-8 and Bcl-2 expression. Compared with blank control group and negative control group at P1-3 BMSCs on E16 transplantation, both caspase-8 and Bcl-2 in mRNA and protein levels were significantly decreased in experimental group with a statistical significance on E19 (Figure 5D,E and F).

\section{Discussion}

Neural tube defects (NTDs) are a class of congenital malformations with serious consequences. The worldwide incidence of NTDs ranges from 1.0 to 10.0 per 1000 births [16]. Almost equal frequencies between two major categories: anencephaly and spina bifida aperta [17]. The exact mechanism of NTDs is unknown up to now, a variety of genetic and environmental factors are implicated [18]. Besides, The development in nervous system is a complex process that involves the proliferation, differentiation or apoptosis of neurons, glial cells synapse, neural networks and nerve nuclei in three-dimensional building. Apoptosis is also called programmed cell death, which is a special form of cell death distinguished from necrosis. It is subject to its internal organization of certain cells in the gene-regulation and active biological phenomenon suicide, which is widespread in embryonic development, organ or tissue morphogenesis [19]. The regulation from the nervous system, neurotrophic factors secreted by cells and other mechanisms restrains excessive cell apoptosis. If any element of these mechanisms is damaged, it will result in excessive cells apoptosis to the occurrence of spina bifida aperta [20].

Mammalian embryonic development is a highly dynamic process involving a high degree of embryonic cellular division, growth, and differentiation which can be influenced by disrupted signaling pathways (Wells, Lee, McCallum, Perstin, \& Harper, 2010). STAT3 regulates multiple embryonic development pathways that play an important role in the abnormal embryonic development which may be altered by VPA [21]. In addition, STAT3 modulates gene transcription in response to intermediate factors, such as EGF, IL-6, Bcl-2 and caspase-8, whereas these factors regulate cell proliferation, survival, and apoptosis during embryogenesis (Hirano, Ishihara, \& Hibi, 2000; Liu et al., 2010; Zhong et al., 1994). In vivo, STAT3 is an inactive non-phosphorylated monomer. Once stimulated by cytokines, STAT3 will phosphorylate, and then undergo homologous or heterodimerization. Then, enter the nucleus and combine with specific nucleic acid sequences to start the expression of various downstream target genes [22]. From our results, pSTAT3 in mRNA and protein levels were significantly increased in experimental group from E18 to E20, and E19 reached the peak. The expression of STAT3 mRNA and protein levels in experimental group gradually decreased with embryonic development between E18 and E19, E19 dropped down to bottom. These evidences suggested that STAT3 was phosphorylated in the process of transplantation and converted to PSTAT3 on E19, which could activate the expression of downstream target genes and possibly promote the trans-differentiation of BMSCs.

BMSCs has a strong self-renewal capacity and pluripotency, which could be induced to differentiate into bone cells, cartilage cells, fat cells, endothelial cells, myocytes, nerve cells and other cell types in particular environment [23]. The BMSCs transplantation of SBA can repair a variety of neuronal and bone abnormalities. In addition, BMSCs are easily obtained, operated, cultured in vitro and purified, which can 
be passaged for many times to maintain differentiation capacity, but no matching and immune rejection. It has recent studies showed that BMSCs can survive in the spinal cord injury and differentiate into neurons and astrocytes to reduce the damage caused by the loss of nerve function [24]. Therefore, BMSCs can be used as tissue-engineering cells, and BMSCs transplantation for the treatment of SBA is expected to a most promising method of nervous system malformations. The purposes of surgical repair on spinal cord for treatment early in utero of rat fetuses with SBA are to reduce further aggravation of malformations. As our study showed that BMSCs combined with STAT3 overexpression plasmid transfection can remarkably improve cell purity and survival rates. Cell transplantation from P1 to P3 on E16 with medium injection in experimental group could obtain better therapeutic effect than blank control group and negative control group. Studies have shown that STAT3 pathway may play an important role in the migration and differentiation of BMSCs [25]. STAT3 interacts with other signaling pathways, such as MAPK and PI32K, which is expressed and activated during the development of nervous system, and participates in the differentiation of stem cells in neural tube epithelium [26]. Peripherin, as a neuron specific intermediate filament protein, which has a STAT3 binding site in the promoter and is expressed in all peripheral neurons, sensory and motor neurons [27]. STAT3 plays a transcriptional activity under the action of LIF and IL-6, suggesting that it also has regulatory function on peripheral neuron [28]. Aberg M et al [29] observe that STAT3 activation can specifically mediate the directional differentiation of type II astrocytes. All these results suggest that JAK/STAT signaling pathway activated by cytokine is an important mechanism in determining the direction of cell differentiation during mammalian development. Although STAT3 is one of the most important regulatory genes in the development of nervous system, the survival rate of embryos after gene knockout is very low in vivo, which limits STAT3 research on the regulation process and molecular mechanism [30].

In recent years, the culture success of BMSCs in vitro has provided a good experimental model for further study of the important biological characteristics, such as proliferation, differentiation, migration and so on [31]. Therefore, the effect of STAT3 partial activation on the proliferation and differentiation of BMSCs in vitro has not been reported. JAK/STAT3 signaling pathway is closely related to maintain pluripotency and undifferentiated state of embryonic stem cells [32]. Under LIF treatment, stem cells overexpressing negative STAT3 will lose the ability of self-renewal and differentiate, revealing that STAT3 is closely related to the proliferation of neural stem cells [33]. Our results showed that OD value in experimental group was the highest at 8 hours after transplantation, and then gradually decreased, which was significantly different from blank control group and negative control group. On the one hand, transfection of STAT3 overexpression plasmid can promote the transformation at 8 hours after transplantation between BMSCs and neural cells; on the other hand, it may increase survival and proliferation of cells. In addition, some studies show that STAT3 plays an important role in the differentiation of neural precursor cells in vivo and interacts with other signaling pathways, such as MAPK and caspase-8, and then participates in neuromodulatory cytokine (NF), leukemia inhibitory factor (LIF), ciliary neurotrophic factor (CNTF), CT1 (cardiotrophin), Oncostatin $\mathrm{M}$ to regulate the differentiation and fate of neural precursor cells [34]. In this process, SOCS family, as STAT3 inhibitors, acts as a negative feedback protein to inhibit JAK activity. Moreover, STAT3 has a binding site in the promoter of glial fibrillary acidic protein (GFAP), 
and activates the transcription of GFAP during the differentiation of cortical precursor cells into glial cells induced by CNTF. Therefore, it is believed that STAT3 is closely related to the differentiation of astrocytes [35]. Further studies showed that STAT3 combined with Smad1 and p300 formed a complex named as STAT3/Smad1/p300 through synergistic treatment of LIF and bone morphogenetic protein 2 (BMP-2), which mediated the activation of NSE, NF and Nestin promoter, and induced primary embryonic neural precursor cells to differentiate into astrocytes. The above-mentioned evidence proved that STAT3 mainly induces the differentiation of neural stem cells into astrocytes [36]. Our results also showed that the mRNA and protein levels of GFAP, NSE, NF and Nestin in experimental group were significantly higher than that in blank control group and negative control group. It indicated that STAT3 was involved in the differentiation of MSCs into osteocytes, muscular and neuroglia cells. STAT3 signaling pathway and MAP kinase mediate neurite growth induced by LIF in PC12 cells. The activation of STAT3 can negatively regulate the neurite elongation of PC12 cells dependent on Ras-MAPK pathway, and promote the contraction of sympathetic neuron dendrites in vitro [37]. It is demonstrated that STAT3 can regulate neurite growth, which is consistent with the results of our studies.

Meanwhile, caspase- 8 plays the role of initiator in the caspase cascade and is a key molecule in death receptor-induced apoptotic pathways. It has been shown that Caspase-8 is essential for Fas-mediated apoptotic signaling [38]. Activated Caspase-8 cleaves and activates downstream caspases, thereby committing the cell to apoptosis. Caspase-8 and Bcl-2 also activates another apoptotic pathway, which, through processing of Bid, mediates release of cytochrome $\mathrm{C}$ from mitochondria and activation of Caspase-9. And, in addition to Fas, Caspase-8 is involved in apoptotic signaling via other death receptors, such as tumor necrosis factor receptor type 1 (TNFR1), death receptor 3 (DR3) and TNF-related apoptosis-inducing ligand (TRAIL) receptors. Clearly, Caspase-8 and Bcl-2 are important mediators for a wide range of apoptotic pathways [39]. In our study, compared with blank control group and negative control group, both Caspase-8 and Bcl-2 in mRNA and protein levels were significantly decreased in experimental group on E19. The results mean that BMSCs transplantation after STAT3 overexpression plasmid transfection can reduce apoptosis in the spinal column. The Caspase-8 and bcl-2-mediated signaling pathway, as apoptosis functions during BMSCs transplantation after STAT3 overexpression plasmid transfection, requires further elucidation.

\section{Conclusion}

In conclusion, our results support the notion that transfection of recombinant lentivirus mediated STAT3 overexpression plasmid with BMSCs can improve the efficiency of transforming into neural cells and provide new seed cells for the treatment of congenital spina bifida aperta.

\section{Abbreviations}

STAT3: signal transducer and activator of transcription-3

BMSCs: bone mesenchymal Stem Cells 
SBA: spina bifida aperta

E20: 20-day pregnant

NTDs: neural tube defects

GFP: green fluorescent protein

RA: retinoic acid

NF: neuromodulatory cytokine

OD: optical density

CCK-8: Cell Counting Kit-8

LIF: leukemia inhibitory factor

CNTF: ciliary neurotrophic factor

GFAP: glial fibrillary acidic protein

TNFR1: tumor necrosis factor receptor type 1

TRAIL: TNF-related apoptosis-inducing ligand

MAPK:mitogen-activated protein kinase.

\section{Declarations}

\section{Availability of data and materials}

The datasets used and/or analyzed during the current study are available from the corresponding author on reasonable request.

\section{Acknowledgements}

Thank you for the support by Heilongjiang Provincial Universities, projects of basic scientific research business funds, from Education Department of Heilongjiang Province in 2019.

\section{Funding}

This study was supported by Heilongjiang Provincial Universities, projects of basic scientific research business funds, from Education Department of Heilongjiang Province in 2019 (2019-KYYWF-0332), which played crucial roles in research design, collection, analysis, and interpretation of data.

\section{Author information}




\section{Affiliations}

Department of Pediatrics, The First Affiliated Hospital of Harbin Medical University, 23 Youzheng Street, Nangang District, Harbin, 150001 Heilongjiang, PR China

Ming-yu Jiang, Ji-cheng Dai \& Ming-yong Ren

Department of Pediatrics, The Fourth Affiliated Hospital of Harbin Medical University, Harbin, Heilongjiang, PR China;

Ming-ying Yin

\section{Contributions}

Jiang Ming-yu drafted the manuscript. Ming-yong Ren significantly contributed to all the figure designs used throughout the manuscript. Yin Ming-ying critically reviewed the manuscript. Dai Ji-cheng conceived the concept of work. All authors read and approved the final manuscript.

\section{Corresponding author}

Correspondence to Ming-yu Jiang, M.D

\section{Ethics declarations}

\section{Ethics approval and consent to participate}

The study is approved by the ethical committee of Harbin medical University of technology committee.

\section{Consent for publication}

Not applicable

\section{Competing interests}

No competing interests, financial or otherwise, declared by the authors.

\section{References}

1. Jeelani Y, Mosich GM, McComb JG. Closed neural tube defects in children with caudal regression. Childs Nerv Syst. 2013;29(9):1451-7.

2. Aoulad Fares D, Schalekamp-Timmermans S, Nawrot TS, Steegers-Theunissen RPM. Preconception telomere length as a novel maternal biomarker to assess the risk of spina bifida in the offspring. Birth Defects Res. 2020,15;112(9):645-651.

3. Wang S, Ji D, Yang Q, Li M, Ma Z, Zhang S, et al. NEFLb impairs early nervous system development via regulation of neuron apoptosis in zebrafish. J Cell Physiol. 2019;234(7):11208-11218. 
4. Geden MJ, Romero SE, Deshmukh M. Apoptosis versus axon pruning: Molecular intersection of two distinct pathways for axon degeneration. Neurosci Res. 2019;139:3-8.

5. Abdallah MA, Ahmed KM, Recio-Restrepo MV, Khalid M, Yeddi A, Abu-Heija A, et al. Pneumococcal Meningitis Complicated by Spinal Cord Dysfunction and Acute Polyradiculopathy. Ochsner J. 2020;20(2):219-221.

6. Kitada M, Dezawa M. Induction system of neural and muscle lineage cells from bone marrow stromal cells; a new strategy for tissue reconstruction in degenerative diseases. Histol Histopathol. 2009;24(5):631-42.

7. Muench MO, Barcena A. Stem cell transplantation in the fetus. Cancer Control. 2004; 11: 105-18.

8. Golchin A, Farahany TZ, Khojasteh A, Soleimanifar F, Ardeshirylajimi A. The Clinical Trials of Mesenchymal Stem Cell Therapy in Skin Diseases: An Update and Concise Review. Curr Stem Cell Res Ther. 2019;14(1):22-33.

9. Myneni VD, McClain-Caldwell I, Martin D, Vitale-Cross L, Marko K, Firriolo JM, et al. Mesenchymal stromal cells from infants with simple polydactyly modulate immune responses more efficiently than adult mesenchymal stromal cells. Cytotherapy. 2019;21(2):148-161.

10. Hsia HC, Hutti JE, Baldwin AS. Cytosolic DNA Promotes Signal Transducer and Activator of Transcription 3 (STAT3) Phosphorylation by TANK-binding Kinase 1 (TBK1) to Restrain STAT3 Activity. J Biol Chem. 2017;292(13):5405-5417.

11. Al-Massri KF, Ahmed LA, El-Abhar HS. Mesenchymal stem cells therapy enhances the efficacy of pregabalin and prevents its motor impairment in paclitaxel-induced neuropathy in rats: Role of Notch1 receptor and JAK/STAT signaling pathway. Behav Brain Res. 2019;360:303-311.

12. Mossahebi-Mohammadi M, Quan M, Zhang JS, Li X. FGF Signaling Pathway: A Key Regulator of Stem Cell Pluripotency. Front Cell Dev Biol. 2020,18;8:79.

13. Fritsch M, Günther SD, Schwarzer R, Albert MC, Schorn F, Werthenbach JP, et al. Caspase-8 is the molecular switch for apoptosis, necroptosis and pyroptosis. Nature. 2019;575(7784):683-687.

14. Schwarzer R, Laurien L, Pasparakis M. New insights into the regulation of apoptosis, necroptosis, and pyroptosis by receptor interacting protein kinase 1 and caspase-8. Curr Opin Cell Biol. 2020;63:186-193.

15. Yin F, Zhou H, Fang Y, Li C, He Y, Yu L, et al. Astragaloside IV alleviates ischemia reperfusion-induced apoptosis by inhibiting the activation of key factors in death receptor pathway and mitochondrial pathway. J Ethnopharmacol. 2020,10;248:112319.

16. Auffret M, Cottin J, Vial T, Cucherat M. Clomiphene citrate and neural tube defects: a meta-analysis of controlled observational studies. BJOG. 2019;126(9):1127-1133.

17. Zou J, Wang F, Yang $X$, Wang $H$, Niswander L, Zhang T, et al. Association between rare variants in specific functional pathways and human neural tube defects multiple subphenotypes. Neural Dev. 2020,10;15(1):8.

18. Zohn IE. Mouse Models of Neural Tube Defects. Adv Exp Med Biol. 2020;1236:39-64. 
19. Zhang L, Dong Y, Wang W, Zhao T, Huang T, Khan A, et al. Ethionine Suppresses Mitochondria Autophagy and Induces Apoptosis via Activation of Reactive Oxygen Species in Neural Tube Defects. Front Neurol. 2020,7;11:242.

20. Wei X, Cao S, Ma W, Zhang C, Gu H, Liu D, et al. Intra-Amniotic Delivery of CRMP4 siRNA Improves Mesenchymal Stem Cell Therapy in a Rat Spina Bifida Model. Mol Ther Nucleic Acids. 2020,5;20:502517.

21. Li H, Zhang Z, Gao C, Wu S, Duan Q, Wu H, et al. Combination chemotherapy of valproic acid (VPA) and gemcitabine regulates STAT3/Bmi1 pathway to differentially potentiate the motility of pancreatic cancer cells. Cell Biosci. 2019,18;9:50.

22. Tummers B, Green DR. Caspase-8: regulating life and death. Immunol Rev. 2017;277(1):76-89.

23. An JH, Li FP, He P, Chen JS, Cai ZG, Liu SR, et al. Characteristics of Mesenchymal Stem Cells Isolated from the Bone Marrow of Red Pandas. Zoology (Jena). 2020;140:125775.

24. Mukhamedshina YO, Gracheva OA, Mukhutdinova DM, Chelyshev YA, Rizvanov AA. Mesenchymal stem cells and the neuronal microenvironment in the area of spinal cord injury. Neural Regen Res. 2019;14(2):227-237.

25. Jin W. Role of JAK/STAT3 Signaling in the Regulation of Metastasis, the Transition of Cancer Stem Cells, and Chemoresistance of Cancer by Epithelial-Mesenchymal Transition. Cells. 2020,15;9(1):217.

26. MuhChyi C, Juliandi B, Matsuda T, Nakashima K. Epigenetic regulation of neural stem cell fate during corticogenesis. Int J Dev Neurosci. 2013;31(6):424-33.

27. Lee N, Neitzel KL, Devlin BK, MacLennan AJ. STAT3 phosphorylation in injured axons before sensory and motor neuron nuclei: potential role for STAT3 as a retrograde signaling transcription factor. $\mathrm{J}$ Comp Neurol. 2004,5;474(4):535-45.

28. Gao S, Durstine JL, Koh HJ, Carver WE, Frizzell N, Carson JA. Acute myotube protein synthesis regulation by IL-6-related cytokines. Am J Physiol Cell Physiol. 2017,1;313(5):C487-C500.

29. Aberg MA, Ryttsén F, Hellgren G, Lindell K, Rosengren LE, MacLennan AJ, et al. Selective introduction of antisense oligonucleotides into single adult CNS progenitor cells using electroporation demonstrates the requirement of STAT3 activation for CNTF-induced gliogenesis. Mol Cell Neurosci. 2001;17(3):426-43.

30. Su Y, Chen Z, Du H, Liu R, Wang W, Li H, et al. Silencing miR-21 induces polarization of astrocytes to the A2 phenotype and improves the formation of synapses by targeting glypican 6 via the signal transducer and activator of transcription-3 pathway after acute ischemic spinal cord injury. FASEB J. 2019;33(10):10859-10871.

31. Fathy M, Okabe M, M Othman E, Saad Eldien HM, Yoshida T. Preconditioning of Adipose-Derived Mesenchymal Stem-Like Cells with Eugenol Potentiates Their Migration and Proliferation In Vitro and Therapeutic Abilities in Rat Hepatic Fibrosis. Molecules. 2020,26;25(9):2020.

32. Brick RM, Sun AX, Tuan RS. Neurotrophically Induced Mesenchymal Progenitor Cells Derived from Induced Pluripotent Stem Cells Enhance Neuritogenesis via Neurotrophin and Cytokine Production. Stem Cells Transl Med. 2018;7(1):45-58. 
33. Nicolaidou V, Wong MM, Redpath AN, Ersek A, Baban DF, Williams LM, et al. Monocytes induce STAT3 activation in human mesenchymal stem cells to promote osteoblast formation. PLoS One. 2012;7(7):e39871.

34. Cosenza M, Civallero M, Fiorcari S, Pozzi S, Marcheselli L, Bari A, et al. The histone deacetylase inhibitor romidepsin synergizes with lenalidomide and enhances tumor cell death in T-cell lymphoma cell lines. Cancer Biol Ther. 2016,2;17(10):1094-1106.

35. Wu M, Song D, Li H, Yang Y, Ma X, Deng S, et al. Negative regulators of STAT3 signaling pathway in cancers. Cancer Manag Res. 2019,29;11:4957-4969.

36. Nakashima K, Yanagisawa M, Arakawa H, Kimura N, Hisatsune T, Kawabata M, et al. Synergistic signaling in fetal brain by STAT3-Smad1 complex bridged by p300. Science. 1999,16;284(5413):47982.

37. März P, Ozbek S, Fischer M, Voltz N, Otten U, Rose-John S. Differential response of neuronal cells to a fusion protein of ciliary neurotrophic factor/soluble CNTF-receptor and leukemia inhibitory factor. Eur J Biochem.2002;269(12):3023-31.

38. Kober AM, Legewie S, Pforr C, Fricker N, Eils R, Krammer PH, et al. Caspase-8 activity has an essential role in CD95/Fas-mediated MAPK activation. Cell Death Dis. 2011,6;2(10):e212.

39. Inman GJ, Allday MJ. Apoptosis induced by TGF-beta 1 in Burkitt's lymphoma cells is caspase 8 dependent but is death receptor independent. J Immunol. 2000,1;165(5):2500-10.

\section{Tables}

Due to technical limitations, table 1 is only available as a download in the Supplemental Files section.

\section{Figures}



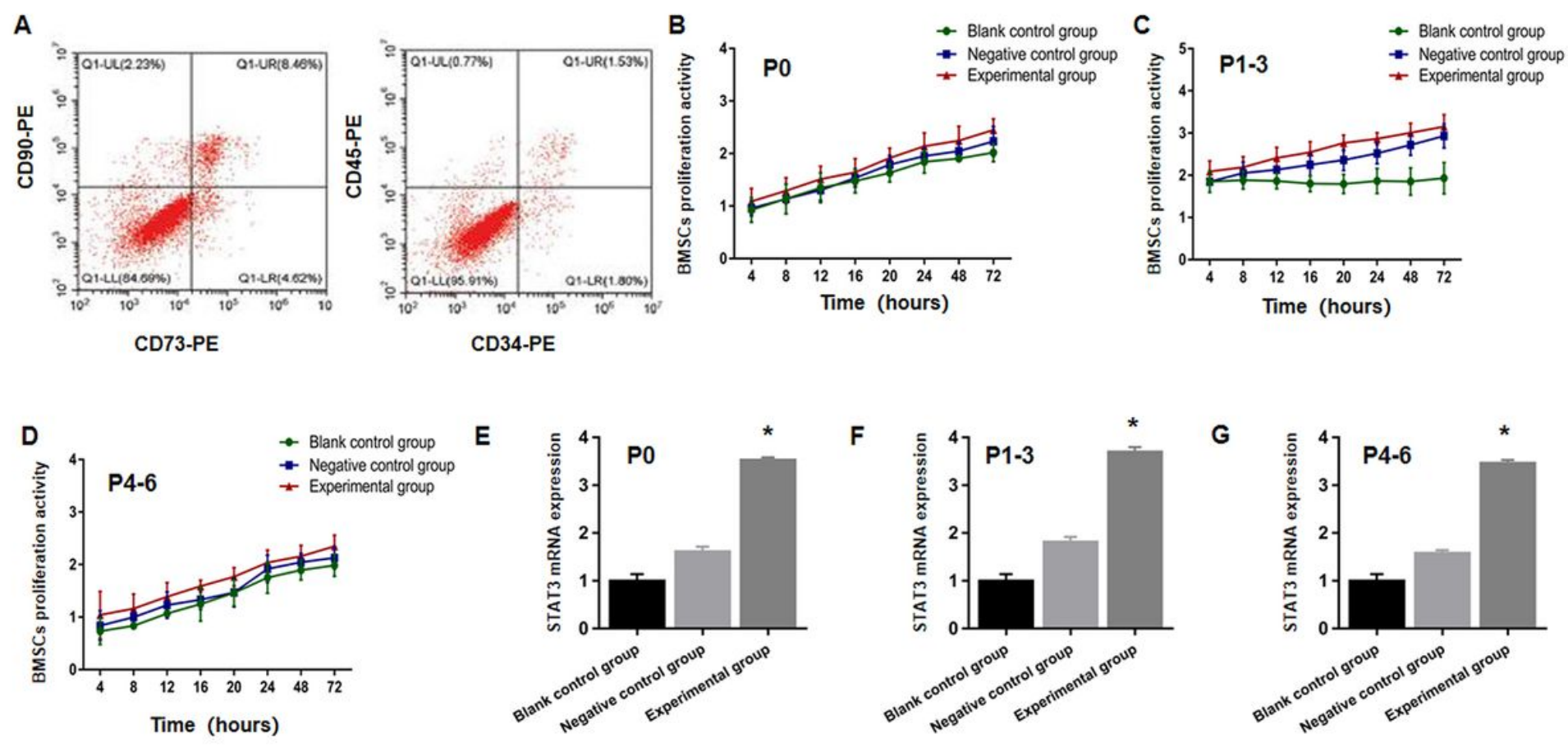

Figure 1

STAT3 plasmid transfected BMSCs were successfully identificated. (A) The expressions of CD34, CD45, CD73 and CD90 from BMSCs were detected using flow cytometry. BMSCs proliferation activity at P0 (B), P1-3 (C), P4-6 (D) were determined by CCK-8 test in blank control group, negative control group and experimental group before transplantation. STAT3 mRNA expression at P0 (E), P1-3 (F), P4-6 (G) BMSCs in experimental group increased more statistically than that in blank control group and negative control group. ${ }^{*} \mathrm{P}<0.05$ versus blank control group + negative control group. 
A

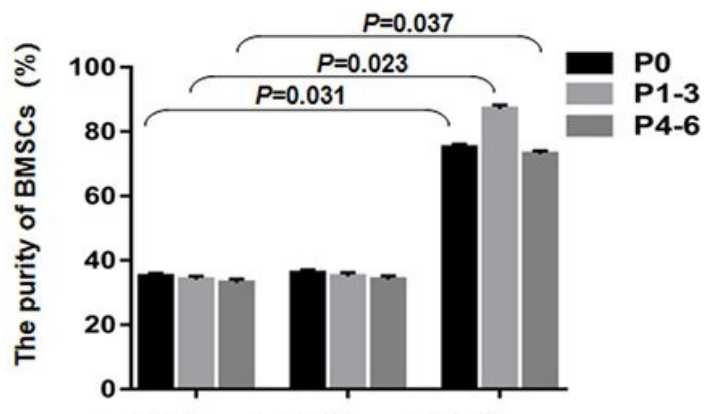

Blank control group Negative control group $_{\text {Experimental group }}$

C

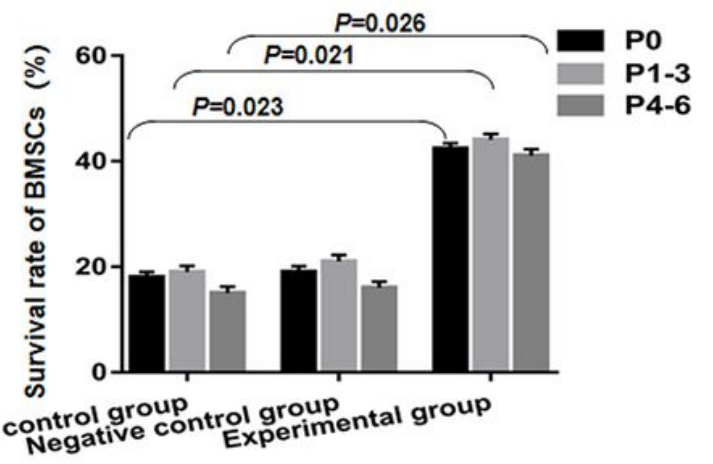

B

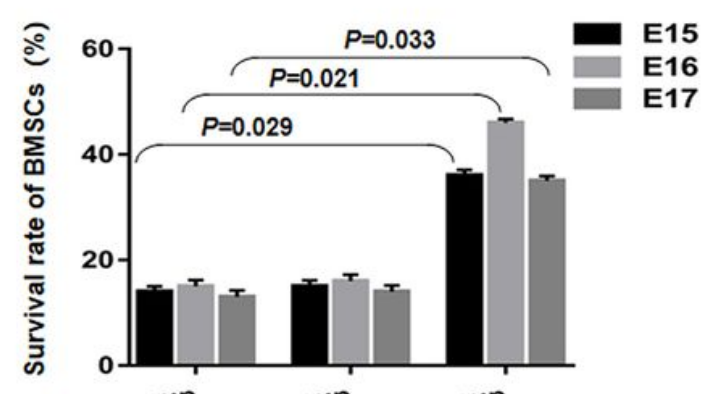

Blank control group Negative control group $_{\text {Experimental group }}$

D

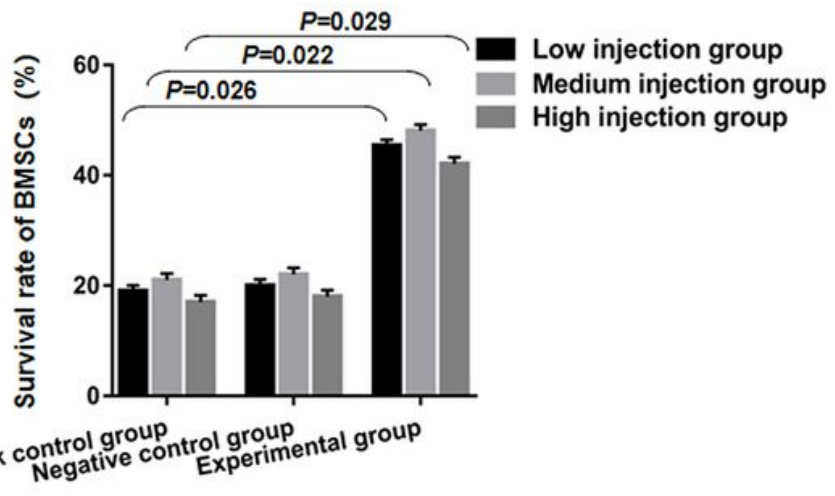

Figure 2

BMSCs combined with STAT3 overexpression plasmid transfection can improve the success rate of transplantation (A) The purity of BMSCs in experimental group was statistically significant, compared with blank control group and negative control group. (B) The overall survival rates of BMSCs in experimental group was found to be a little higher than that of blank control group and negative control group from E15 to E17, and statistically significant. (C) BMSCs survival was found to be notable increase at P0, P1-3 and P4-6 compared with blank control group and negative control group, again the differences reached a statistical significance. (D) Medium injection group was remarkably different from the change that occurred in low injection group and high injection group on E19 fetuses after P1-3 BMSCs transplantation, which reached a statistical significance.
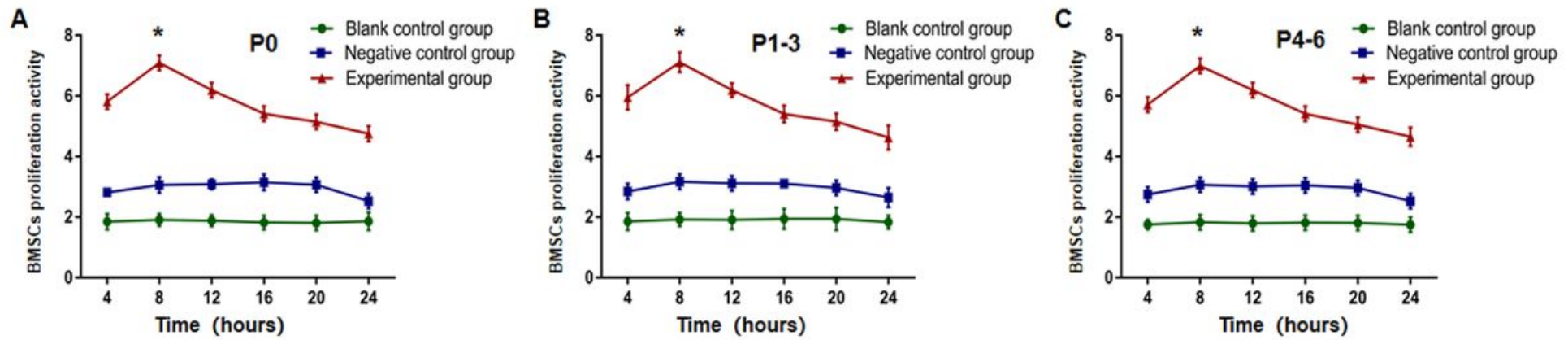

Figure 3 
OD value from $\mathrm{P} 0-6$ BMSCs in experimental group was the highest at 8 hours after E16 transplantation by CCK-8 test. OD value from (A) P0, (B) P1-3 and (C) P4-6 in experimental group was the highest at 8 hours after E16 transplantation, and then gradually decreased, which was statistically significant. * $P<0.05$ versus blank control group + negative control group.

A

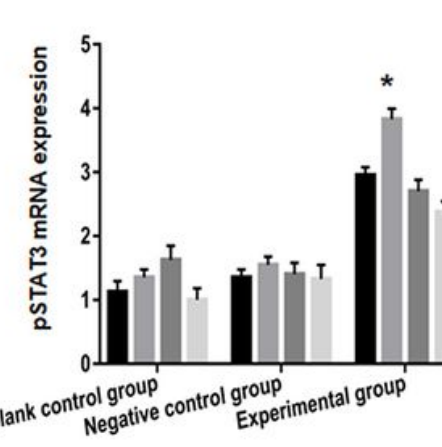

D

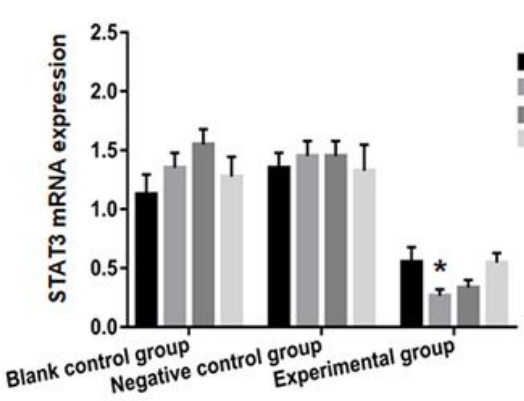

B

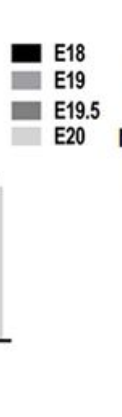

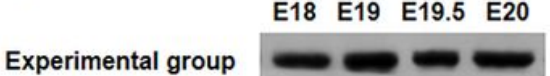

Negative control group

Blank control group

GAPDH
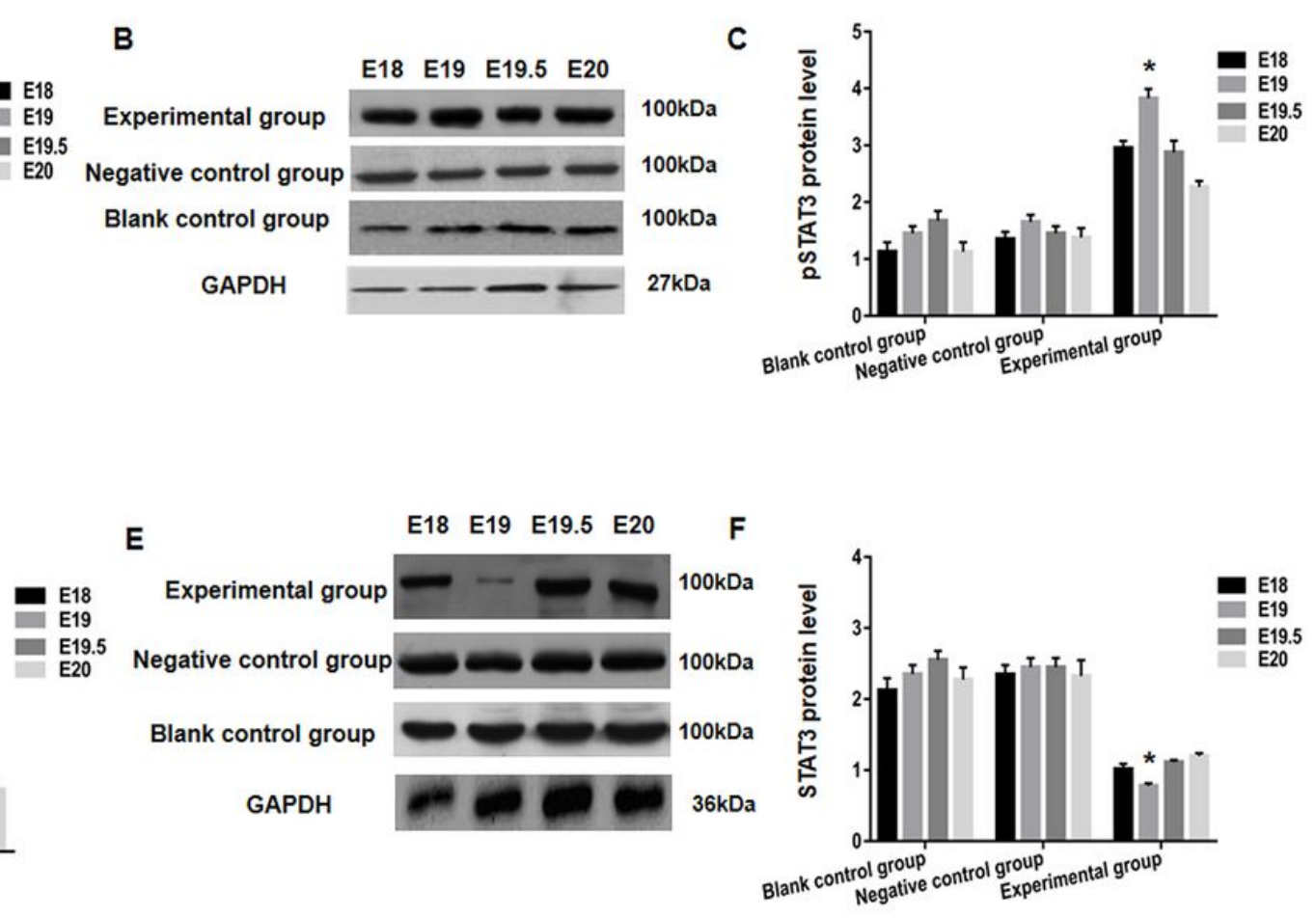

Figure 4

STAT3 and pSTAT3 expression in spinal cord of each group after BMSCs transplantation using RT-PCR and western blot analysis. pSTAT3 mRNA expression was detected using RT-qPCR (A), and protein expression using western blot analysis (B and C). Both pSTAT3 mRNA and protein levels were significantly increased in experimental group from E18 to E20, and E19 reached the peak. In addition, expression of STAT3 mRNA was analysed by RT-qPCR (D). Protein level using western blot (E and F). The expression of STAT3 mRNA and protein levels in experimental group gradually decreased with embryonic development between E18 and E19, E19 dropped down to bottom. ${ }^{*} \mathrm{P}<0.05$ versus blank control group + negative control group. 


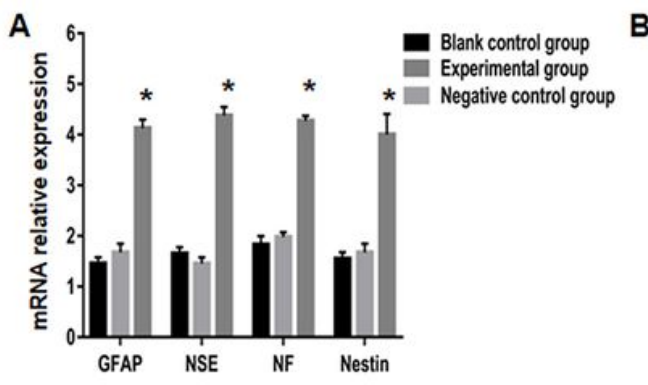

B
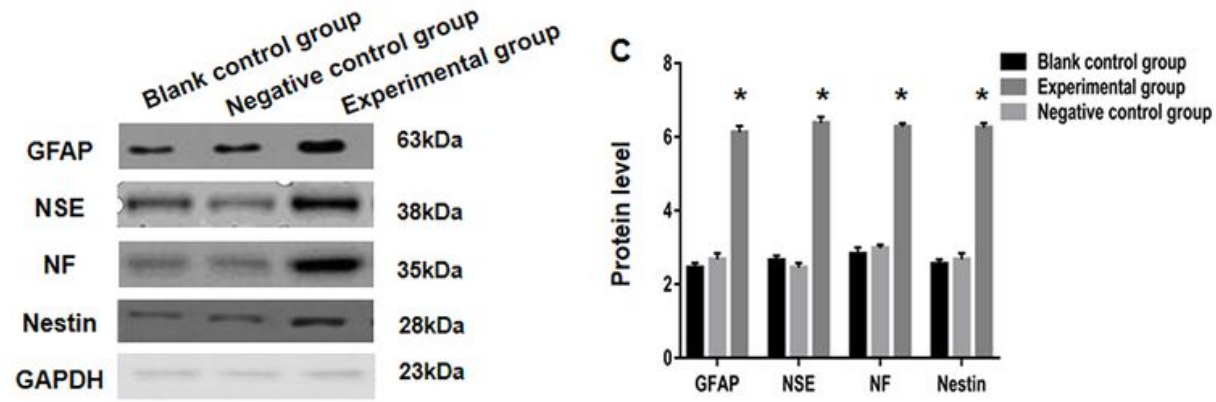

。

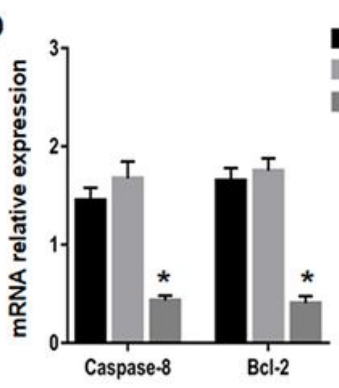

$\mathbf{F}$

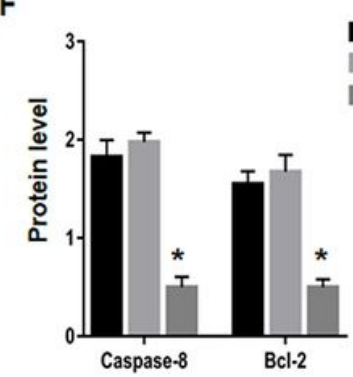

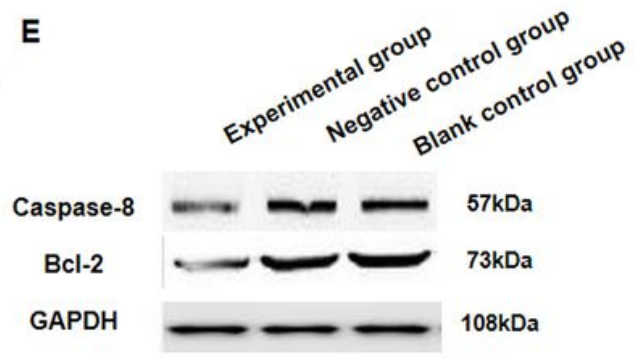

Figure 5

Nerve markers (GFAP, NSE, NF and Nestin) and apoptosis related factors (Caspase 8 and Bcl-2) expressions were tested by the method of real-time PCR and Western blot. (A) Expressions of GFAP, NSE, $\mathrm{NF}$ and Nestin in blank control group,negative control group and experimental group were examined using RT-qPCR, and GFAP, NSE, NF and Nestin protein expression using western blot analysis (B and C). Relative expression of apoptosis related factors (Caspase 8 and Bcl-2) mRNA expression using RT-qPCR (D) and protein expression using western blot analysis ( $E$ and $F$ ) was determined. ${ }^{*} P<0.05$ versus blank control group + negative control group.

\section{Supplementary Files}

This is a list of supplementary files associated with this preprint. Click to download.

- Table.pdf

- data.xls 